\title{
Quantum Fuzzy Logic and Time
}

\author{
Shiro Ishikawa', Kohshi Kikuchi' ${ }^{2}$ \\ ${ }^{1}$ Department of Mathematics, Faculty of Science and Technology, Keio University, Yokohama, Japan \\ ${ }^{2}$ Faculty of Liberal Arts, National Institute of Technology, Kumamoto College, Koshi, Kumamoto, Japan \\ Email: ishikawa@math.keio.ac.jp, kikuchi@kumamoto-nct.ac.jp
}

How to cite this paper: Ishikawa, S. and Kikuchi, K. (2021) Quantum Fuzzy Logic and Time. Journal of Applied Mathematics and Physics, 9, 2609-2622.

https://doi.org/10.4236/jamp.2021.911168

Received: September 30, 2021

Accepted: November 2, 2021

Published: November 5, 2021

Copyright $\odot 2021$ by author(s) and Scientific Research Publishing Inc. This work is licensed under the Creative Commons Attribution International License (CC BY 4.0).

http://creativecommons.org/licenses/by/4.0/ (c) (i) Open Access

\begin{abstract}
Recently we proposed the linguistic Copenhagen interpretation (or, quantum language, measurement theory), which has a great power to describe both classical and quantum systems. Thus we think that quantum language can be viewed as the language of science. Further, we showed that certain logic (called quantum fuzzy logic) works in quantum language. In general, it is said that logic and time do not go well together. Then, the purpose of this paper is to show that quantum fuzzy logic works well with time. That is, quantum fuzzy logic has the advantage of being able to clearly distinguish between implication and causality. In fact, we will show the contraposition of the proposition "If no one is scolded, no one will study" (or the negation of "John is always hungry") can be written in quantum fuzzy logic. However, "time" in everyday language has various aspects (e.g., tense, subjective time). Therefore, it is not possible to understand all of the "time" of everyday language by the "time" of quantum language.
\end{abstract}

\section{Keywords}

Quantum Language, Linguistic Copenhagen Interpretation, Quantum Fuzzy Logic, Time

\section{Introduction}

Recently, in refs. [1]-[6], we proposed the linguistic Copenhagen interpretation (or, quantum language, measurement theory), which has a great linguistic power to describe both classical and quantum systems. Thus we think that quantum language can be viewed as the language of science. As seen in Figure 1 below, roughly speaking, QL has the following four aspects, that is,

$\left(A_{1}\right)$ (7): the linguistic turn of quantum mechanics (cf. refs. [7] [8] [9] [10]);

$\left(\mathrm{A}_{2}\right)$ (8): the dualistic turn of statistics (cf. refs. [11]-[16]); 


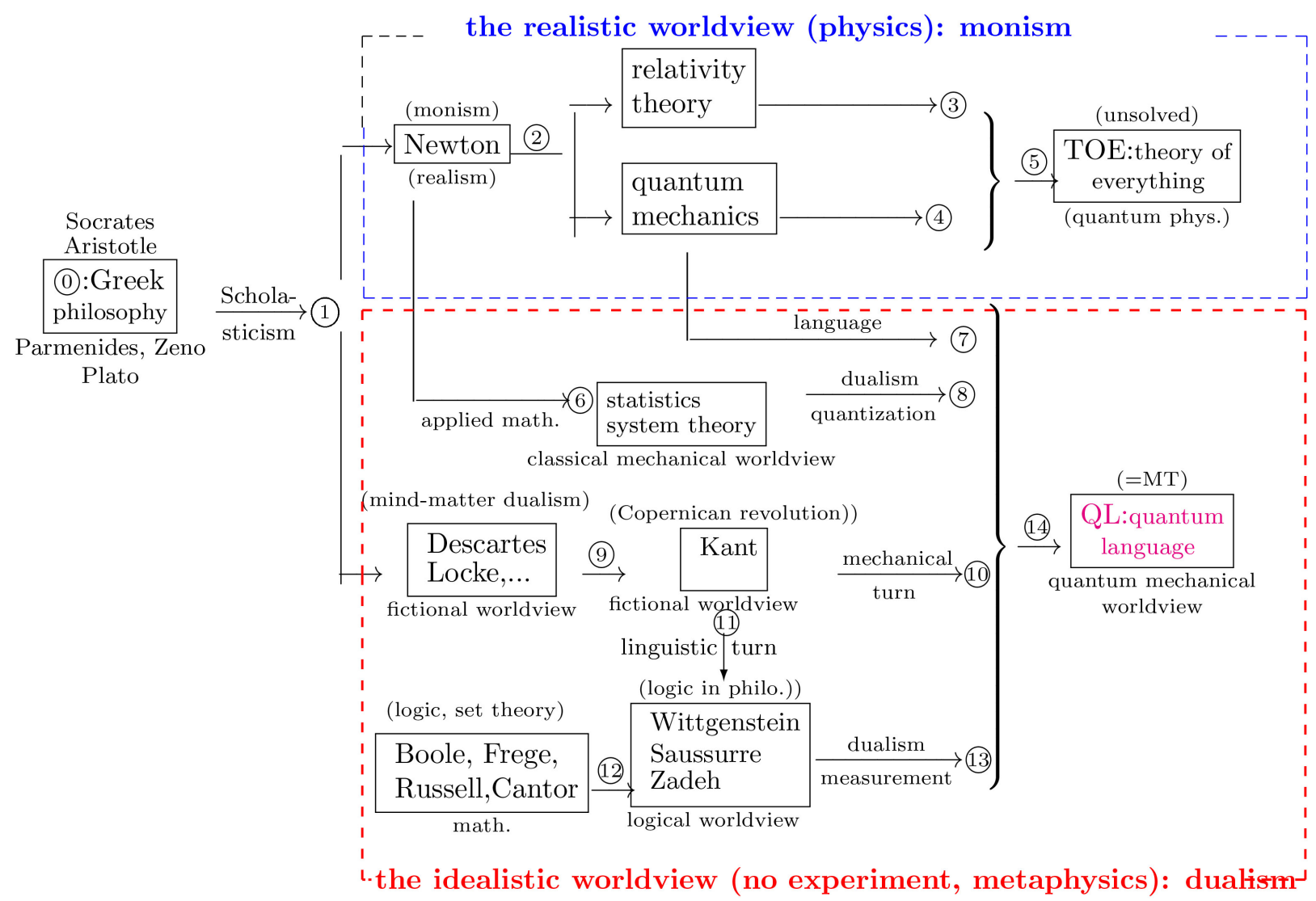

Figure 1. The history of the world-descriptions.

$\left(\mathrm{A}_{3}\right)$ (10): the scientific turn of Descartes = Kant philosophy (cf. refs. [17] [18] [19]);

$\left(\mathrm{A}_{4}\right)$ (13): the logical aspect (cf. refs. [20] [21] [22]).

Thus the location of QL in the history of the world-descriptions is as in Figure 1 (cf. ref. [17] [22] [23]):

The purpose of this paper is to study the logical aspect [13) + (14)] of QL. Although it is generally said that logic and time do not go well together, many researchers have attempted to incorporate time into logic (e.g. ref. [24]). In particular, Pnueli's work (cf. [25]) is highly regarded in the field of computing, and he was awarded the 1997 Turing Award for this achievement. It is natural to consider that mechanics and time series go hand in hand. Therefore, we can expect that quantum fuzzy logic (in [13 + (14)]) and time series are also compatible. In fact, this paper shows that quantum fuzzy logic is as closely related to time as it is to quantum mechanics. However, "time" in everyday language has various aspects (e.g., tense, subjective time). Therefore, it is not possible to understand all of the "time" of everyday language by the "time" of quantum language.

\section{Elementary Review of Classical QL}

In this section, we shall review quantum language (i.e., the linguistic Copenhagen interpretation of quantum mechanics, or measurement theory), which has 
the following form:

(B)

(quantum mechanical worldview)

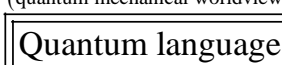

$=\underset{\text { (causality) }}{\text { Axiom } 2}+\underset{\text { (measurement) }}{\text { Axiom } 1}+\frac{\text { linguistic (Copenhagen) interpretation }}{\text { (how to use Axioms } 1 \text { and } 2 \text { ) }}$

QL is classified as follows

$\int\left(\mathrm{C}_{1}\right)$ : quantum $\mathrm{QL}$ for quantum system,

(C)

(which is essentially the same as quantum mechanics)

$\left(\mathrm{C}_{2}\right)$ : classical QL for classical system,

(which may be viewed as statistics with a measurement concept)

It is usual to discuss the above two simultaneously (cf. refs. [5] [6] [8]). However, in this paper, we will devote ourselves to only classical QL. That is because we think that classical QL is easy to understand for readers who are not familiar with quantum theory. We do not want the difficulty of the mathematics ( $c f$. refs. [26] [27] [28]) to hinder the spread of QL.

Let $\Omega$ be a state space, i.e., compact space. An element $\omega$ in $\Omega$ is called a state. And let $C(\Omega)$ be the commutative $C^{*}$-algebra, i.e., the space of all complex-valued continuous functions on $\Omega$.

Definition 1. [Observable, Image observable] According to the noted idea ( $c f$. refs. [29] [30]), an observable $\mathrm{O}=(X, \mathcal{P}(X), G)$ in $C(\Omega)$ is defined as follows:

1) $X$ is a finite set, $\mathcal{P}(X) \quad\left(=2^{X}\right.$, the power set of $\left.X\right)$.

2) $G$ is a mapping from $\mathcal{P}(X)$ to $C(\Omega)$ satisfying: a): for every $\Xi \in \mathcal{P}(X)$, $G(\Xi)$ is a non-negative element in $C(\Omega)$ such that $0 \leq G(\Xi) \leq I, \mathrm{~b})$ : $G(\varnothing)=0$ and $G(X)=I$, where 0 and $I$ are the 0 -element and the identity in $C(\Omega)$ respectively. c): [additivity]

$$
G\left(\Xi_{1}\right)+G\left(\Xi_{2}\right)=G\left(\Xi_{1} \cup \Xi_{2}\right)
$$

for all $\Xi_{1}, \Xi_{2} \in \mathcal{P}(X)$ such that $\Xi_{1} \cap \Xi_{2}=\varnothing$.

If $G(\Xi)=G(\Xi)^{2} \quad(\forall \Xi \in \mathcal{P}(X))$, then $\mathrm{O}=(X, \mathcal{P}(X), G)$ in $C(\Omega)$ is a projective observable (or, crisp observable). Also, $\mathrm{O}=(X, \mathcal{P}(X), G)$ in $C(\Omega)$ is also called an $X$-valued observable. We will devote ourselves to binary (i.e., $\{T, F\}$-valued) observables in most of the cases in this paper. Let $Y$ be a finite set, and let $\Theta: X \rightarrow Y$ be a map. Then, $\Theta(\mathrm{O})=\left(Y, \mathcal{G}, G\left(\Theta^{-1}(\cdot)\right)\right)$ in $C(\Omega)$ is also observable in $C(\Omega)$ (which is called an image observable).

With any classical system $S$, a commutative $C^{*}$-algebra $C(\Omega)$ can be associated in which the measurement theory (B) of that system can be formulated. A state of the system $S$ is represented by an element $\omega(\in \Omega)$ and an observable is represented by an observable $\mathrm{O}=(X, \mathcal{P}(X), G)$ in $C(\Omega)$. Also, the measurement of the observable $\mathrm{O}$ for the system $S$ with the state $\omega_{0}$ is denoted by $\mathrm{M}_{C(\Omega)}\left(\mathrm{O}=(X, \mathcal{P}(X), G), S_{\left[\omega_{0}\right]}\right)$. An observer can obtain a measured value $x(\in X)$ by the measurement $\mathrm{M}\left(\mathrm{O}, S_{\left[\omega_{0}\right]}\right)$. 
The Axiom 1 presented below is a kind of mathematical generalization of Born's probabilistic interpretation of quantum mechanics. And thus, it is a statement without reality.

Axiom 1. [Measurement]. The probability that a measured value $x(\in X)$ obtained by the measurement $\mathrm{M}_{C(\Omega)}\left(\mathrm{O}=(X, \mathcal{P}(X), G), S_{\left[\omega_{0}\right]}\right)$ is given by $[G(\{x\})]\left(\omega_{0}\right)$.

Next, we explain Axiom 2 in (B).

Let $\left(\mathbb{T} \equiv\left\{t_{0}, t_{1}, \cdots, t_{n}\right\}, \leq\right)$ be a (finite) tree-like semi-ordered set with the root $t_{0}$. Let $\Pi: \mathbb{T} \backslash\left\{t_{0}\right\} \rightarrow \mathbb{T}$ is the parent map, that is, $\Pi(t)=t^{\prime}$ is defined such that

$$
t^{\prime} \leq t, \quad t^{\prime} \neq t, \quad \text { “ } t \text { " } \leq t \text { ” implies that } t^{\prime \prime} \leq t^{\prime}
$$

This $\left(\mathbb{T} \equiv\left\{t_{0}, t_{1}, \cdots, t_{n}\right\}, \leq\right)$ is also written by $\left(\mathbb{T} \equiv\left\{t_{0}, t_{1}, \cdots, t_{n}\right\}, \Pi: \mathbb{T} \backslash\left\{t_{0}\right\} \rightarrow \mathbb{T}\right)$.

For example see Figure 2 below, in which we see the root $t_{0}$, the parent map: $\Pi\left(t_{3}\right)=\Pi\left(t_{4}\right)=t_{2}, \Pi\left(t_{2}\right)=\Pi\left(t_{5}\right)=t_{1}, \Pi\left(t_{1}\right)=\Pi\left(t_{6}\right)=\Pi\left(t_{7}\right)=t_{0}$.

Let $\left(\mathbb{T} \equiv\left\{t_{0}, t_{1}, \cdots, t_{n}\right\}, \Pi: \mathbb{T} \backslash\left\{t_{0}\right\} \rightarrow \mathbb{T}\right)$ be a tree-like semi-ordered set with the root $t_{0}$. For each $t \in T \backslash\left\{t_{0}\right\}$, define the homomorphism.

$\Phi_{\Pi(t), t}: C\left(\Omega_{t}\right) \rightarrow C\left(\Omega_{\Pi(t)}\right) \quad$ (see, for example, Figure 3 ), which is characterized as a continuous map $\Phi_{\Pi(t), t}: \Omega_{\Pi(t)} \rightarrow \Omega_{t}$ such that

$$
\left[\Phi_{\Pi(t), t} f_{2}\right]\left(\omega_{1}\right)=f_{2}\left(\phi_{\Pi(t), t}\left(\omega_{1}\right)\right) \quad\left(\forall f_{2} \in C\left(\Omega_{t}\right), \forall \omega_{1} \in \Omega_{\Pi(t)}\right)
$$

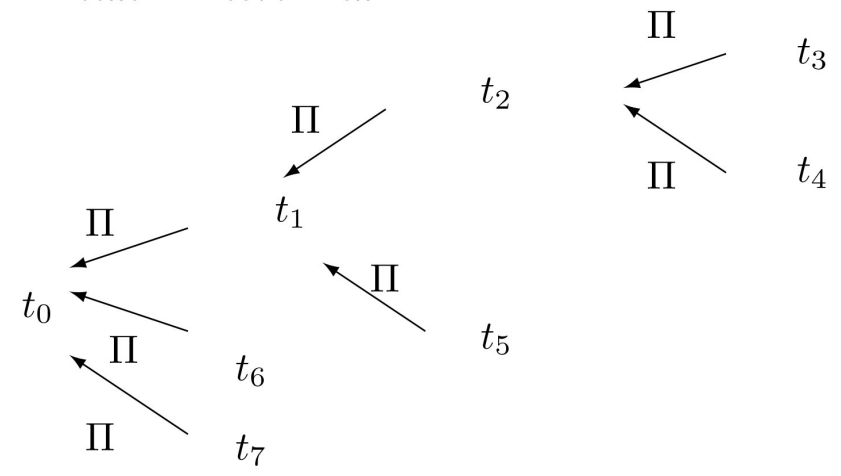

Figure 2. Tree: $\left(T=\left\{t_{0}, t_{1}, \cdots, t_{7}\right\}, \Pi: T \backslash\left\{t_{0}\right\} \rightarrow T\right)$.

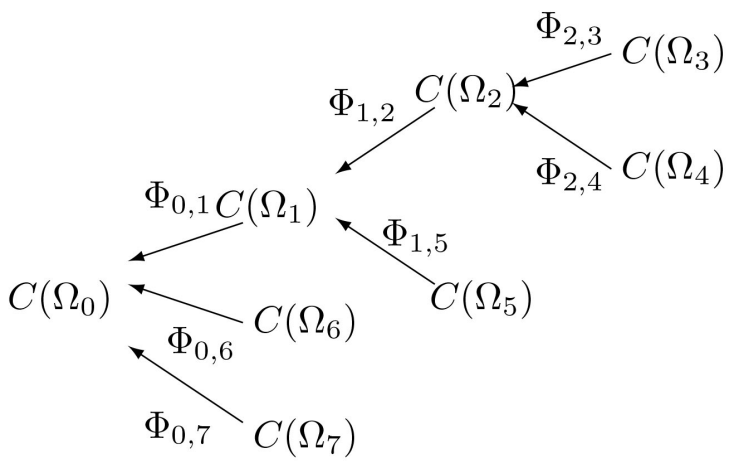

Figure 3. Homomorphism $\Phi_{\Pi(t), t}: C\left(\Omega_{t}\right) \rightarrow C\left(\Omega_{\Pi(t)}\right)$. 
Now we can propose Axiom 2 (i.e., causality). (For details, see ref. [6].)

Axiom 2. [Causality]; Let $\left(\mathbb{T} \equiv\left\{t_{0}, t_{1}, \cdots, t_{n}\right\}, \leq\right)$ be a (finite) tree-like semiordered set with the root $t_{0}$. Causality is represented by $\left\{\right.$ homomorphism $\left.\Phi_{\Pi(t), t}: C\left(\Omega_{t}\right) \rightarrow C\left(\Omega_{\Pi(t)}\right): t \in \mathbb{T} \backslash\left\{t_{0}\right\}\right\}$.

Remark 2. [The linguistic Copenhagen interpretation] Since the linguistic Copenhagen interpretation is the manual to use Axioms 1 and 2, it consists of many rules ( $c f$. refs. [6] [22] [23]). However, for the purposes of this paper, it is sufficient to focus only on the following.

$\left(D_{1}\right)$ Only one measurement is permitted, and thus, the state after a measurement is non-sense. Thus, we proposed the new formulation of projection postulate (i.e., wavefunction collapse) ( $c$ f. ref. [9]).

$\left(D_{2}\right)$ Time should be represented by a tree-like semi-ordered set $\left(\mathbb{T} \equiv\left\{t_{0}, t_{1}, \cdots, t_{n}\right\}, \leq\right)$ in Axiom 2.

$\left(D_{3}\right)$ The Heisenberg picture $\mathrm{M}_{C\left(\Omega_{0}\right)}\left(\Phi_{t_{0}, t_{1}} \mathrm{O}_{1}, S_{\left[\omega_{t_{0}}\right]}\right)$ is used and the Schrödinger picture $\mathrm{M}_{C\left(\Omega_{t_{1}}\right)}\left(\mathrm{O}_{1}, S_{\left[\phi_{t_{0}, t_{1}}\left(\omega_{0}\right)\right]}\right)$ is not.

$\left(\mathrm{D}_{4}\right)$ The subjective time (i.e., observer's time, tense) does not exist. (cf. Leibniz $=$ Clarke correspondence in ref. [19].)

$\left(D_{5}\right)$ The measurer cannot measure himself. Thus, the assertions: "I think", "I am", etc. are not scientific. Therefore, "I think, therefore I am" is not a scientific proposition.

We need the following definition for the above $\left(D_{1}\right)$.

Definition 3. [(i): Quasi-product observable, quasi-product measurement]: Let $\mathrm{O}_{i}=\left(X_{i}, \mathcal{P}\left(X_{i}\right), G_{i}\right) \quad(i=1,2, \cdots, N)$ be commutative observables in $C(\Omega)$. Define the quasi-product observable

$$
\begin{aligned}
\times_{i=1,2, \cdots, n}^{q p} \mathrm{O}_{i}= & \left(\times_{i=1}^{n} X_{i}, \mathcal{P}\left(\times_{i=1}^{n} X_{i}\right), \times_{i=1,2, \cdots, n}^{q p} G_{i}\right) \text { such that } \\
& {\left[\begin{array}{c}
q p \\
\underset{i=1,2, \cdots, n}{\times}
\end{array} G_{i}\right]\left(X_{1} \times X_{2} \times \cdots \times X_{j-1} \times \Xi_{j} \times X_{j+1} \times \cdots \times X_{n}\right)=G_{j}\left(\Xi_{j}\right) } \\
& \left(\forall \Xi_{j} \in \mathcal{P}\left(X_{j}\right), j=1,2, \cdots, n\right)
\end{aligned}
$$

Also, $\mathrm{M}_{C(\Omega)}\left(x_{i=1,2, \cdots, n}^{q p} \mathrm{O}_{i}=\left(x_{i=1}^{n} X_{i}, \mathcal{P}\left(x_{i=1}^{n} X_{i}\right), x_{i=1,2, \cdots, n}^{q p} G_{i}\right), S_{\left[\omega_{0}\right]}\right)$ is called the quasi-product measurement of $\mathrm{M}_{C(\Omega)}\left(\mathrm{O}_{i}=\left(X_{i}, \mathcal{P}\left(X_{i}\right), G_{i}\right), S_{\left[\omega_{0}\right]}\right)(i=1,2, \cdots, n)$.

[(ii): Tensor $C^{*}$-algebra, tensor product observable, tensor quasi-product measurement]: Let $\mathrm{O}_{i}=\left(X_{i}, \mathcal{P}\left(X_{i}\right), G_{i}\right)$ be observables in $C(\Omega)_{i}$, $(i=1,2, \cdots, n)$. Define a tensor product observable $\otimes_{i=1,2, \cdots, n} \mathrm{O}_{i}=\left(x_{i=1}^{n} X_{i}, \mathcal{P}\left(x_{i=1}^{n} X_{i}\right), \otimes_{i=1,2, \cdots, n} G_{i}\right)$ in the tensor $C^{*}$-algebra $\otimes_{i=1}^{n} C\left(\Omega_{i}\right) \quad\left(=C\left(\times_{i=1}^{n} \Omega_{i}\right)\right)$. such that

$$
\left[\bigotimes_{i=1}^{n} G_{i}\right]\left(\begin{array}{c}
n \\
\times \\
i=1
\end{array} \Xi_{j}\right)=\bigotimes_{i=1}^{n} G_{i}\left(\Xi_{i}\right) \quad\left(\forall \Xi_{i} \in \mathcal{P}\left(X_{i}\right), i=1,2, \cdots, n\right)
$$

Also, $\quad \mathrm{M}_{\otimes_{i=1}^{n} C\left(\Omega_{i}\right)}\left(\otimes_{i=1,2, \cdots, n} \mathrm{O}_{i}=\left(x_{i=1}^{n} X_{i}, \mathcal{P}\left(x_{i=1}^{n} X_{i}\right), \otimes_{i=1,2, \cdots, n} G_{i}\right), S_{\left[\left(\omega_{1}, \omega_{2}, \cdots, \omega_{n}\right)\right]}\right)$ is called a tensor product measurement of $\mathrm{M}_{C\left(\Omega_{i}\right)}\left(\mathrm{O}_{i}=\left(X_{i}, \mathcal{P}\left(X_{i}\right), G_{i}\right), S_{\left[\omega_{i}\right]}\right)$ 
$(i=1,2, \cdots, n)$.

\section{Quantum Fuzzy Logic in Classical QL}

In the previous section, we introduced classical QL, which is the mathematical representation of the quantum mechanical worldview. In this section, we introduce the quantum fuzzy logic in the quantum mechanical worldview. We believe that Wittgenstein's purpose of TLP (in ref. [31]) is to propose the definition of "proposition" (i.e., to define "what we can speak about"). And thus, in ref. [22] (particularly Theorem 16 in ref. [22] is the fundamental theorem in quantum fuzzy logic), we asserted that "quantum fuzzy logic" realized Wittgenstein's dream.

Let's start with the following definition.

Definition 4. [( $T F)$-measurement (=Fuzzy proposition), Fuzzy set (= Membership function)] Let $\mathrm{O}=(\{T, F\}, \mathcal{P}(\{T, F\}), G)$ be a binary observable (or, $(T F)$-observable, $\{T, F\}$-valued observable) in a commutative $C^{*}$-algebra $C(\Omega)$. A measurement $\mathrm{M}_{C(\Omega)}\left(\mathrm{O}, S_{\left[\omega_{0}\right]}\right)$ is called a $(T F)$-measurement, which is also called a fuzzy proposition. Since Axiom 1 says that the probability that a measured value $T$ is obtained by $(T F)$-measurement $\mathrm{M}_{C(\Omega)}\left(\mathrm{O}, S_{\left[\omega_{0}\right]}\right)$ is given by $[G(\{T\})]\left(\omega_{0}\right)$, we say that

$\left(\mathrm{E}_{1}\right)$ a $(T F)$-measurement $\mathrm{M}_{C(\Omega)}\left(\mathrm{O}, S_{\left[\omega_{0}\right]}\right)$ is true with probability $[G(\{T\})]\left(\omega_{0}\right)$.

Or,

$\left(\mathrm{E}_{2}\right) \operatorname{Prob}\left[\mathrm{M}_{C(\Omega)}\left(\mathrm{O}, S_{\left[\omega_{0}\right]}\right) ;\{T\}\right]=[G(\{T\})]\left(\omega_{0}\right)$

Also, $G(\{T\})(\in C(\Omega))$ is called the membership function of $\mathrm{O}$.

Definition 5. [Quantum fuzzy logic symbols $(\neg, \wedge, \vee, \rightarrow))$ ] Let $\mathrm{O}_{i}=\left(\{T, F\}, \mathcal{P}(\{T, F\}), G_{i}\right)$ be binary observables (or, $\{T, F\}$-valued observable) in a commutative $C^{*}$-algebra $C(\Omega),(i=1,2)$. Fix the quasi-product observable $\mathrm{O}_{1} \times^{q p} \mathrm{O}_{2}=\left(\{T, F\}^{2}, \mathcal{P}\left(\{T, F\}^{2}\right), G_{1} \times^{q p} G_{2}\right)$. Consider (TF)-measurement $\mathrm{M}_{C(\Omega)}\left(\mathrm{O}_{i}=\left(\{T, F\}, \mathcal{P}(\{T, F\}), G_{i}\right), S_{\left[\omega_{0}\right]}\right)$ (which is abbreviated as $P_{i}$ ) in a $C^{*}$-algebra $C(\Omega)$. Put $\mu_{i}(\Xi)=[G(\{T\})]\left(\omega_{0}\right) \quad(\Xi \in\{T, F\}, i=0,1,2)$, and $\left(\times_{i=1,2}^{q p} \mu_{i}\right)\left(\Xi_{1} \times \Xi_{2}\right)=\left[\left(G_{1} \times{ }^{q p} G_{2}\right)\left(\Xi_{1} \times \Xi_{2}\right)\right]\left(\omega_{0}\right) \quad\left(\Xi_{1}, \Xi_{2} \in 2\{T, F\}\right)$.

(i; Negation): Put $i=1,2$. Remembering the image observable in Definition 1, let's define $\neg \mathrm{M}_{C(\Omega)}\left(\mathrm{O}_{i}, S_{\left[\omega_{0}\right]}\right)$ such that

$$
\left.\neg \mathrm{M}_{C(\Omega)}\left(\mathrm{O}_{i}, S_{\left[\omega_{0}\right]}\right)=\mathrm{M}_{C(\Omega)}(\pi\urcorner \mathrm{O}_{i}, S_{\left[\omega_{0}\right]}\right)
$$

where the map $\pi\urcorner\{T, F\} \rightarrow\{T, F\} \quad$ is defined by $\pi\urcorner(T)=F, \quad \pi\urcorner(F)=T$. Clearly, it holds that $\operatorname{Prob}\left[\neg \mathrm{M}_{C(\Omega)}\left(\mathrm{O}_{i}, S_{\left[\omega_{0}\right]}\right) ;\{T\}\right]=\left[G_{i}(\{F\})\right]\left(\omega_{0}\right)=\mu_{i}(\{F\})$.

(ii; And): Define $\mathrm{M}_{C(\Omega)}\left(\mathrm{O}_{1}, S_{\left[\omega_{0}\right]}\right) \wedge \mathrm{M}_{C(\Omega)}\left(\mathrm{O}_{2}, S_{\left[\omega_{0}\right]}\right)$ such that

$$
\mathrm{M}_{C(\Omega)}\left(\mathrm{O}_{1}, S_{\left[\omega_{0}\right]}\right) \wedge \mathrm{M}_{C(\Omega)}\left(\mathrm{O}_{2}, S_{\left[\omega_{0}\right]}\right)=\mathrm{M}_{C(\Omega)}\left(\pi^{\wedge}\left(\mathrm{O}_{1} \times{ }^{q p} \mathrm{O}_{2}\right), S_{\left[\omega_{0}\right]}\right)
$$

where $\pi^{\wedge}:\{T, F\}^{2} \rightarrow\{T, F\}$ is defined by $\pi^{\wedge}(T, T)=T$, $\pi^{\wedge}(T, F)=\pi^{\wedge}(F, T)=\pi^{\wedge}(T, F)=F$.

It holds that 


$$
\begin{aligned}
& \operatorname{Prob}\left[\mathrm{M}_{C(\Omega)}\left(\mathrm{O}_{1}, S_{\left[\omega_{0}\right]}\right) \wedge \mathrm{M}_{C(\Omega)}\left(\mathrm{O}_{2}, S_{\left[\omega_{0}\right]}\right) ;\{T\}\right] \\
& =\left[\left(G_{1} \times^{q p} G_{2}\right)\left(\left(\pi^{\wedge}\right)^{-1}(\{T\})\right)\right]\left(\omega_{0}\right)=\left(\mu_{1} \times^{q p} \mu_{2}\right)(\{(T, T)\})
\end{aligned} .
$$

(iii; Or): Define $\mathrm{M}_{C(\Omega)}\left(\mathrm{O}_{1}, S_{\left[\omega_{0}\right]}\right) \vee \mathrm{M}_{C(\Omega)}\left(\mathrm{O}_{2}, S_{\left[\omega_{0}\right]}\right)$ such that

$$
\mathrm{M}_{C(\Omega)}\left(\mathrm{O}_{1}, S_{\left[\omega_{0}\right]}\right) \vee \mathrm{M}_{C(\Omega)}\left(\mathrm{O}_{2}, S_{\left[\omega_{0}\right]}\right)=\mathrm{M}_{C(\Omega)}\left(\pi^{\vee}\left(\mathrm{O}_{1} \times{ }^{q p} \mathrm{O}_{2}\right), S_{\left[\omega_{0}\right]}\right)
$$

where $\pi^{\vee}:\{T, F\}^{2} \rightarrow\{T, F\}$ is defined by $\pi^{\vee}(T, T)=\pi^{\vee}(T, F)=\pi^{\vee}(F, T)=T$, $\pi^{\vee}(F, F)=F$.

It holds that

$$
\begin{aligned}
& \operatorname{Prob}\left[\mathrm{M}_{C(\Omega)}\left(\mathrm{O}_{1}, S_{\left[\omega_{0}\right]}\right) \vee \mathrm{M}_{C(\Omega)}\left(\mathrm{O}_{2}, S_{\left[\omega_{0}\right]}\right) ;\{T\}\right] \\
& =\left[\left(G_{1} \times^{q p} G_{2}\right)\left(\left(\pi^{\vee}\right)^{-1}(\{T\})\right)\right]\left(\omega_{0}\right)=\left(\mu_{1} \times^{q p} \mu_{2}\right)(\{(T, T),(T, F),(F, T)\})
\end{aligned} .
$$

(iv; Implication): Define $\mathrm{M}_{C(\Omega)}\left(\mathrm{O}_{1}, S_{\left[\omega_{0}\right]}\right) \rightarrow \mathrm{M}_{C(\Omega)}\left(\mathrm{O}_{2}, S_{\left[\omega_{0}\right]}\right)$ such that

$$
\mathrm{M}_{C(\Omega)}\left(\mathrm{O}_{1}, S_{\left[\omega_{0}\right]}\right) \rightarrow \mathrm{M}_{C(\Omega)}\left(\mathrm{O}_{2}, S_{\left[\omega_{0}\right]}\right)=\mathrm{M}_{C(\Omega)}\left(\pi^{\rightarrow}\left(\mathrm{O}_{1} \times{ }^{q p} \mathrm{O}_{2}\right), S_{\left[\omega_{0}\right]}\right)
$$

where $\pi^{\rightarrow}:\{T, F\}^{2} \rightarrow\{T, F\}$ is defined by $\pi^{\rightarrow}(T, T)=\pi^{\rightarrow}(F, T)=\pi^{\rightarrow}(F, F)=1, \pi^{\rightarrow}(T, F)=F$.

It holds that

$$
\begin{aligned}
& \operatorname{Prob}\left[\mathrm{M}_{C(\Omega)}\left(\mathrm{O}_{1}, S_{\left[\omega_{0}\right]}\right) \rightarrow \mathrm{M}_{C(\Omega)}\left(\mathrm{O}_{2}, S_{\left[\omega_{0}\right]}\right) ;\{T\}\right] \\
& =\left[\left(G_{1} \times{ }^{q p} G_{2}\right)\left(\left(\pi^{\rightarrow}\right)^{-1}(\{T\})\right)\right]\left(\omega_{0}\right)=\left(\mu_{1} \times^{q p} \mu_{2}\right)(\{(T, T),(F, F),(F, T)\})
\end{aligned} .
$$

Example 6. As with mathematical logic, truth tables are useful in quantum fuzzy logic. Concerning $\mathrm{M}_{C(\Omega)}\left(\mathrm{O}_{1}, S_{\left[\omega_{0}\right]}\right)$ and $\mathrm{M}_{C(\Omega)}\left(\mathrm{O}_{2}, S_{\left[\omega_{0}\right]}\right)$, we get the following QL version of the truth table (i.e., Table 1).

\begin{tabular}{|c|c|c|c|c|}
\hline $\begin{array}{c}\mathrm{M}_{C(\Omega)}\left(\mathrm{O}_{1}, \mathrm{~S}_{\left[\omega_{0}\right]}\right) \\
\left(=P_{1}\right)\end{array}$ & $\begin{array}{c}\mathrm{M}_{C(\Omega)}\left(\mathrm{O}_{2}, S_{\left[\omega_{0}\right]}\right) \\
\left(=P_{2}\right)\end{array}$ & $\begin{array}{l}\text { probability: } \\
p=\times_{i=1,2}^{q p} \mu_{i}\end{array}$ & {$\left[P_{1} \rightarrow P_{2}\right]$} & {$\left[\neg P_{2} \rightarrow \neg P_{1}\right]$} \\
\hline$T$ & $T$ & $p_{12}=\times_{i=1,2}^{q p} \mu_{i}(\{(T, T)\})$ & $T$ & $T$ \\
\hline$T$ & $F$ & $p_{1 \overline{2}}=x_{i=1,2}^{q p} \mu_{i}(\{(T, F)\})$ & $F$ & $F$ \\
\hline$F$ & $T$ & $p_{\overline{1} 2}=x_{i=1,2}^{q p} \mu_{i}(\{(F, T)\})$ & $T$ & $T$ \\
\hline$F$ & $F$ & $p_{\overline{1} \overline{2}}=x_{i=1,2}^{q p} \mu_{i}(\{(F, F)\})$ & $T$ & $T$ \\
\hline
\end{tabular}

For example, we see that

$$
\operatorname{Prob}\left[\left[P_{1} \rightarrow P_{2}\right] ;\{T\}\right]=p_{12}+p_{\overline{1} 2}+p_{\overline{1} \overline{2}}
$$

\section{Time in Quantum Fuzzy Logic}

\subsection{Parallel Times Series; John Is Always Hungry}

Let $\Omega\left(=\Omega_{1}=\Omega_{2}\right)$ be a compact state space, each element of which is assumed

Table 1. Probabilistic truth table (Elementary propositions $\left.\mathrm{M}_{C(\Omega)}\left(\mathrm{O}_{1}, S_{\left[\omega_{0}\right]}\right)\left(=P_{1}\right), \mathrm{M}_{C(\Omega)}\left(\mathrm{O}_{2}, S_{\left[\omega_{0}\right]}\right)\left(=P_{2}\right)\right)$. 
to represent the state of the human mind. Consider the membership function $h: \Omega \rightarrow[0,1]$ such that

$h(\omega)=\theta \Leftrightarrow \omega$ is the state of human mind with intensity hunger of Degree $\theta$

which is usually interpreted as follows. That is, $h: \omega \rightarrow[0,1]$ is defined by the following:

(F) When asked, "Does the person with state $\omega$ feel hungry?", $100 h(\omega)$ percent said "yes".

This is essentially the same as the probabilistic interpretation of membership functions (cf. refs. [20] [23]).

Define the observables $\mathrm{O}=(\{T, F\}, \mathcal{P}(\{T, F\}), H)$ be a binary observable (or, $(T F)$-observable, $\{T, F\}$-valued observable) in a commutative $C^{*}$-algebra $C(\Omega)$ such that

$$
H(\{T\})=h, \quad H(\{F\})=1-h
$$

Let $\omega^{0}(\in \Omega)$ be John's mind state. We have the $(T F)$-measurement (i.e., $\mathrm{M}_{C(\Omega)}\left(\mathrm{O}=(\{T, F\}, \mathcal{P}(\{T, F\}), H), S_{\left[\omega^{0}\right]}\right)$, which can be identified with the following proposition:

(G) John is hungry.

That is, we see

$\left(\mathrm{H}_{1}\right)$ a $(T F)$-measurement $\mathrm{M}_{C(\Omega)}\left(\mathrm{O}, S_{\left[\omega^{0}\right]}\right)$ is true with probability $[(G(\{T\}))]\left(\omega^{0}\right)$.

Or,

$\left(\mathrm{H}_{2}\right) \operatorname{Prob}\left[\mathrm{M}_{C(\Omega)}\left(\mathrm{O}, S_{\left[\omega_{0}\right]}\right) ;\{T\}\right]=[(G(\{T\}))]\left(\omega^{0}\right)$

Next, we will study "John is always hungry". For this, we must consider $\mathrm{M}_{C(\Omega)}\left(\mathrm{O}=(\{T, F\}, \mathcal{P}(\{T, F\}), H), S_{\left[\omega_{i}^{0}\right]}\right)(i=1,2, \cdots, n)$.

Define the tree-like semi-ordered set $\left(\mathbb{T} \equiv\left\{t_{0}, t_{1}, \cdots, t_{n}\right\}, \Pi: \mathbb{T} \backslash\left\{t_{0}\right\} \rightarrow \mathbb{T}\right)$ such that $t_{0}=\Pi\left(t_{j}\right)(\forall j=1,2, \cdots, n)$. Assume that for each $t\left(\in \mathbb{T} \backslash\left\{t_{0}\right\}\right)$, a $C(\Omega)$ is associated (i.e., $C\left(\Omega_{t}\right)=C(\Omega)$ ), and $C\left(\Omega_{t_{0}}\right)=\otimes_{i=1}^{n} C\left(\Omega_{t_{i}}\right)=C\left(\Omega^{n}\right)$. And, for any $i(=1,2, \cdots, n)$, define a homomorphism $\Phi_{t_{0}, t_{i}}: C(\Omega) \rightarrow C\left(\Omega^{n}\right)$ such that $\phi_{t_{0}, t_{i}}: \Omega^{n} \rightarrow \Omega$ satisfies that

$$
\phi_{t_{0}, t_{i}}\left(\omega_{1}, \omega_{2}, \cdots, \omega_{i}, \cdots, \omega_{n}\right)=\omega_{i} \quad\left(\forall\left(\omega_{1}, \omega_{2}, \cdots, \omega_{i}, \cdots, \omega_{n}\right) \in \Omega^{n}\right)
$$

(See, for example Figure 4)

According to $\left(D_{2}\right)$ in Remark 2 (The linguistic Copenhagen interpretation), we regard the above tree-like semi-ordered set $\left(\mathbb{T} \equiv\left\{t_{0}, t_{1}, \cdots, t_{n}\right\}, \leq\right)$ as time.

Let $\omega_{i}^{0}(\in \Omega)$ be John's mind state at time $t_{i}(i=1,2, \cdots, n)$. Thus, note that, for each $i=1,2, \cdots, n$, the $(T F)$-measurement (i.e., $\mathrm{M}_{C(\Omega)}\left(\mathrm{O}=(\{T, F\}, \mathcal{P}(\{T, F\}), H), S_{\left[\omega_{i}^{0}\right]}\right)$ can be identified with the following proposition:

(I) John is hungry at time $t_{i}$. 


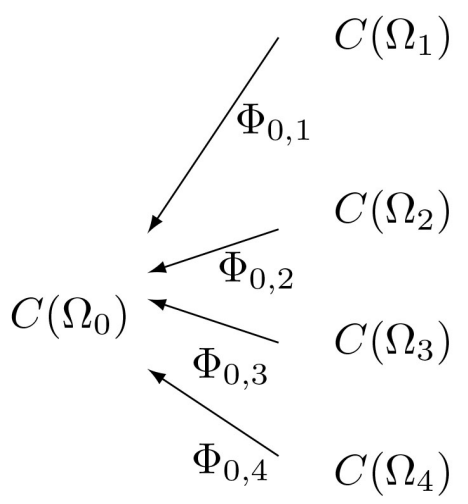

Figure 4. Homomorphism $\Phi_{\Pi(t), t}: C\left(\Omega_{t}\right) \rightarrow C\left(\Omega_{\Pi(t)}\right):(\Pi(t)=0(\forall t=1,2,3,4))$.

Define the tensor product observables $\otimes_{i=1}^{n} \mathrm{O}=\left(\{T, F\}^{n}, \mathcal{P}\left(\{T, F\}^{n}\right), \otimes_{i=1}^{n} H\right)$ in $\otimes_{i=1}^{n} C(\Omega)\left(=C\left(\Omega^{n}\right)\right)$ and consider the measurement $\mathrm{M}_{C\left(\Omega^{n}\right)}\left(\pi^{\wedge} \otimes_{i=1}^{n} \mathrm{O}, S_{\left[\left(\omega_{1}^{0}, \omega_{2}^{0}, \cdots, \omega_{n}^{0}\right)\right]}\right)$. Define the map $\pi^{\wedge}:\{T, F\}^{n} \rightarrow\{T, F\}$ such that

$$
\pi^{\wedge}\left(x_{1}, x_{2}, \cdots, x_{n}\right)= \begin{cases}T & \left(\text { if } x_{1}=x_{2}=\cdots=x_{n}=T\right) \\ F & (\text { otherwise })\end{cases}
$$

Thus, we get the $(T F)$-measurement $\mathrm{M}_{C\left(\Omega^{n}\right)}\left(\pi^{\wedge} \otimes_{i=1}^{n} \mathrm{O}, S_{\left[\left(\omega_{1}^{0}, \omega_{2}^{0}, \cdots, \omega_{n}^{0}\right)\right]}\right)$, which implies that

(J) John is always hungry.

That is, we see

$\left(\mathrm{K}_{1}\right)$ a $(\mathrm{TF})$-measurement the $(T F)$-measurement

$\mathrm{M}_{C\left(\Omega^{n}\right)}\left(\pi^{\wedge} \otimes_{i=1}^{n} \mathrm{O}, S_{\left[\left(\omega_{1}^{0}, \omega_{2}^{0}, \cdots, \omega_{n}^{0}\right)\right]}\right)$

Or,

$\left(\mathrm{K}_{2}\right) \operatorname{Prob}\left[\mathrm{M}_{C\left(\Omega^{n}\right)}\left(\pi^{\wedge} \otimes_{i=1}^{n} \mathrm{O}, S_{\left[\left(\omega_{1}^{0}, \omega_{2}^{0}, \cdots, \omega_{n}^{0}\right)\right]}\right)\right]$ is true with probability $\times_{i=1}^{n}[(H(\{T\}))]\left(\omega_{i}^{0}\right)$

Remark 7 (i): For example, consider the following sentence:

(L) John was hungry yesterday and the day before yesterday.

This sentence (L) has to do with tense. Thus, as seen in Remark 2 (The linguistic Copenhagen interpretation), this is not a meaningful proposition within QL.

(ii): The above parallel time in Figure 4 does not match our daily senses, and the reader may feel uncomfortable. However, this time plays an essential role in the understanding of Hume's problem of induction (cf. ref. [20]). It is rather interesting that there is a gap between our everyday sensory understanding and our scientific understanding. This gap is the reason why Hume's problem of induction has remained unresolved for so long. 


\subsection{If No One Is Scolded, No One Study}

Here let us consider the following proposition:

(M) If no one is scolded, no one will study.

Our present purpose is to rewrite this proposition in quantum fuzzy logic. Let $\Omega\left(=\Omega_{1}=\Omega_{2}\right)$ be a compact state space, each element of which represents the mind of human being. Consider the membership function $g_{1}: \Omega \rightarrow[0,1]$ such that

$$
\begin{aligned}
& g_{1}(\omega)=\theta_{1} \\
& \Leftrightarrow \omega \text { is the state of mind of a person who is scolded } \\
& \quad \text { with at the strength of Degree } \theta_{1}\left(0 \leq \theta_{1} \leq 1\right) .
\end{aligned}
$$

which is usually interpreted as follows.

$(\mathrm{N})$ When asked, "Does the person feel scolded?", $100 g_{1}(\omega)$ out of 100 respond "yes".

This is essentially the same as the probabilistic interpretation of membership functions (cf. refs. [20] [23]).

Further consider the membership function $g_{2}: \Omega \rightarrow[0,1]$ such that

$$
\begin{aligned}
& g_{2}(\omega)=\theta_{2} \\
& \Leftrightarrow \omega \text { is the state of mind of a person who wants to } \\
& \text { study at the strength of Degree } \theta_{2}\left(0 \leq \theta_{2} \leq 1\right) .
\end{aligned}
$$

Also, consider the time evolution

$\Phi_{t_{1} t_{2}}\left(t_{1}<t_{2} ; t_{1}\right.$ is the root $): C(\Omega)\left(=C\left(\Omega_{2}\right)\right) \rightarrow C(\Omega)\left(=C\left(\Omega_{1}\right)\right)$, which is characterized by the continuous map $\phi_{t_{1}, t_{2}}: \Omega\left(=\Omega_{1}\right) \rightarrow \Omega\left(=\Omega_{2}\right)$ such that

$$
\left(\Phi_{t_{1} t_{2}} f_{2}\right)\left(\omega_{1}\right)=f_{2}\left(\phi_{t_{1}, t_{2}}\left(\omega_{1}\right)\right) \quad\left(\forall f_{2} \in C\left(\Omega_{2}\right), \forall \omega_{1} \in \Omega_{1}\right)
$$

which is illustrated in Figure 5 below.

Define the observables $\mathrm{O}_{i}=\left(\{T, F\}, \mathcal{P}(\{T, F\}), G_{i}\right)$ be a binary observable (or, $(T F)$-observable, $\{T, F\}$-valued observable) in a commutative $C^{*}$-algebra $C(\Omega) \quad(i=1,2)$ such that

$$
\left.G_{i}(\{T\})=g_{i}, \quad G_{i}(\{F\})\right)=1-g_{i}
$$

Let $\omega_{0}\left(\in \Omega=\Omega_{1}\right)$ be a state of the system $S$.

Here consider two (TF)-measurements (i.e., fuzzy propositions)

$\mathrm{M}_{C(\Omega)}\left(\mathrm{O}_{1}, S_{\left[\omega_{0}\right]}\right)$ and $\mathrm{M}_{C(\Omega)}\left(\Phi_{t_{1}, t_{2}} \mathrm{O}_{2}, S_{\left[\omega_{0}\right]}\right)$ (which is respectively abbreviated as $\left.P_{i}\right)$. Here, it should be noted that the Heisenberg picture $\mathrm{M}_{C(\Omega)}\left(\Phi_{t_{1}, t_{2}} \mathrm{O}_{2}, S_{\left[\omega_{0}\right]}\right)$ is used and the Schrödinger picture $\mathrm{M}_{C(\Omega)}\left(\mathrm{O}_{2}, S_{\left[\phi_{t_{1}, t_{2}}\left(\omega_{0}\right)\right]}\right)$ is not.

Fix the quasi-product observable $\mathrm{O}_{1} \times{ }^{q p} \Phi_{t_{1}, t_{2}} \mathrm{O}_{2}=\left(\{T, F\}^{2}, \mathcal{P}\left(\{T, F\}^{2}\right), G_{1} \times \times^{q p} \Phi_{t_{1}, t_{2}} G_{2}\right)$ in $C(\Omega)$. And consider quasi-product measurement $\mathrm{M}_{C(\Omega)}\left(\mathrm{O}_{1} \times{ }^{q p} \Phi_{t_{1}, t_{2}} \mathrm{O}_{2}, S_{\left[\omega_{0}\right]}\right)$. Put $\mu_{1}(\Xi)=\left[G_{1}(\Xi)\right]\left(\omega_{0}\right) \quad(\Xi \subseteq\{T, F\}), \quad \mu_{2}(\Xi)=\left[\Phi_{t_{1}, t_{2}} G_{2}(\Xi)\right]\left(\omega_{0}\right)$ $(\Xi \subseteq\{T, F\})$, and $\left(\times_{i=1,2}^{q p} \mu_{i}\right)\left(\Xi_{1} \times \Xi_{2}\right)=\left[\left(G_{1} \times{ }^{q p} \Phi_{t_{1}, t_{2}} G_{2}\right)\left(\Xi_{1} \times \Xi_{2}\right)\right]\left(\omega_{0}\right)$ $\left(\Xi_{1}, \Xi_{2} \subseteq\{T, F\}\right.$ ) (see Table 2). 


$$
C\left(\Omega_{t_{1}}\right) \longleftarrow \Phi_{t_{1}, t_{2}} \longleftarrow C\left(\Omega_{t_{2}}\right)
$$

Figure 5. Homomorphism $\Phi_{t_{1}, t_{2}}: C\left(\Omega_{t_{2}}\right) \rightarrow C\left(\Omega_{t_{1}}\right)$.

Table 2. Probabilistic truth table (Elementary propositions $\left.\mathrm{M}_{C(\Omega)}\left(\mathrm{O}_{1}, S_{\left[\omega_{0}\right]}\right)\left(=P_{1}\right), \quad \mathrm{M}_{C(\Omega)}\left(\Phi_{t_{1}, t_{2}} \mathrm{O}_{2}, S_{\left[\omega_{0}\right]}\right)\left(=P_{2}\right)\right)$.

\begin{tabular}{|c|c|c|c|c|}
\hline $\begin{array}{c}\mathrm{M}_{C(\Omega)}\left(\mathrm{O}_{1}, S_{\left[\omega_{0,}\right]}\right) \\
\left(=P_{1}\right)\end{array}$ & $\begin{array}{c}\mathrm{M}_{C(\Omega)}\left(\Phi_{t_{1}, t_{2}} \mathrm{O}_{2}, S_{\left[\omega_{0}\right]}\right) \\
\left(=P_{2}\right)\end{array}$ & $\begin{array}{l}\text { probability: } \\
p=x_{i=1,2}^{q p} \mu_{i}\end{array}$ & {$\left[\neg P_{1} \rightarrow \neg P_{2}\right]$} & {$\left[P_{2} \rightarrow P_{1}\right]$} \\
\hline$T$ & $T$ & $p_{12}=\times_{i=1,2}^{q p} \mu_{i}(\{(T, T)\})$ & $T$ & $T$ \\
\hline$T$ & $F$ & $p_{1 \overline{2}}=x_{i=1,2}^{q p} \mu_{i}(\{(T, F)\})$ & $F$ & $F$ \\
\hline$F$ & $T$ & $p_{\overline{1} 2}=x_{i=1,2}^{q p} \mu_{i}(\{(F, T)\})$ & $T$ & $T$ \\
\hline$F$ & $F$ & $p_{\overline{1} \overline{2}}=x_{i=1,2}^{q p} \mu_{i}(\{(F, F)\})$ & $T$ & $T$ \\
\hline
\end{tabular}

Therefore we see, by Table 2 , that if

“ $p_{1 \overline{2}}\left(=\times_{i=1,2}^{q p} \mu_{i}(\{(T, F)\})=\left[\left(G_{1} \times{ }^{q p} \Phi_{t_{1}, t_{2}} G_{2}\right)(\{T\} \times\{F\})\right]\left(\omega_{0}\right)\right)=0$ ”, then it holds that

"[ $\left.\neg P_{1} \rightarrow \neg P_{2}\right]$ is true" ( $\approx$ If no one is scolded at time $t_{1}$, then no one study at time $\left.t_{2}\right)$ $\left(\Leftrightarrow\right.$ “[ $\left.P_{2} \rightarrow P_{1}\right]$ is true" $\left(\approx\right.$ If someone is studying at time $t_{2}$, then he was scolded at time $\left.\left.t_{1}\right)\right)$

Remark 8. As seen in Section 3, quantum fuzzy logic (e.g., implication) is produced by measurement (i.e., Axiom 1). On the other hand, causality (and time) arises from Axiom 2. For example, we can see both "implication" and "causality" in the above quasi-product measurement $\mathrm{M}_{C\left(\Omega_{1}\right)}\left(\mathrm{O}_{1} \times{ }^{q p} \Phi_{t_{1}, t_{2}} \mathrm{O}_{2}, S_{\left[\omega_{0}\right]}\right)$. We think the difference between "implication" and "causality" is now clear in quantum language.

Remark 9. Bertrand Russell said in ref. [32] "The Analysis of Mind", p. 223.

- We cannot deny the hypothesis that the world began five minutes ago. which is true since this is a consequence of the linguistic Copenhagen interpretation $\left(\mathrm{D}_{4}\right)$ (cf. ref. [19]). Therefore, the sentence "the world began five minutes ago" is not a proposition in QL.

\section{Conclusions}

It is usual that "logic" is constructed in mathematics (e.g., see ref. [33]). On the other hand, our logic (i.e., quantum fuzzy logic in ref. [22]) is constructed in the quantum language (which has been proposed as the "language of science"). And thus, we can expect that quantum fuzzy logic plays an important role in science. This is the motivation for us to write this paper.

It is generally said that mathematical logic and time are not compatible. We believe this is certain. However, our logic is not mathematical logic, but quantum fuzzy logic and quantum fuzzy logic is a logic born from quantum mechan- 
ics. It is natural to think that mechanics and time are compatible, so there is a reason to expect quantum fuzzy logic and time to be compatible. As this paper shows, it can be said that this expectation was correct to some extent. In fact, in Section 4, we showed the denial of the proposition of "John is always hungry" and the contraposition of the proposition of "If no one is scolded, no one study" in quantum fuzzy logic.

In everyday language, we are free to use tense and subjective time, but in the quantum language (or science), the use of tense and subjective time is forbidden. Quantum language is a scientific language, so it is natural that we cannot use the word "time" unscientifically ( $c f$. Remark 9: Russell's "the world began five minutes ago"). On the other hand, although the parallel time is scientific time, it is interesting that this time has a gap with the nuances of everyday language ( $c f$. Remark 7: Related to Hume's problem of induction).

As a discussion about logic and time within the framework of science, we believe that our proposal is the best. We hope that our proposal will be examined from various points of view.

\section{Conflicts of Interest}

The authors declare no conflicts of interest regarding the publication of this paper.

\section{References}

[1] Ishikawa, S. (1997) Fuzzy Inferences by Algebraic Method. Fuzzy Sets and Systems, 87, 181-200. https://doi.org/10.1016/S0165-0114(96)00035-8 http://www.sciencedirect.com/science/article/pii/S0165011496000358

[2] Ishikawa, S. (1997) A Quantum Mechanical Approach to Fuzzy Theory. Fuzzy Sets and Systems, 90, 277-306. https://doi.org/10.1016/S0165-0114(96)00114-5 https://www.sciencedirect.com/science/article/abs/pii/S0165011496001145

[3] Ishikawa, S. (1998) Fuzzy Logic in Measurements. Fuzzy Sets and Systems, 100, 291-300. https://doi.org/10.1016/S0165-0114(97)00154-1 https://www.sciencedirect.com/science/article/abs/pii/S0165011497001541

[4] Ishikawa, S. (2000) Statistics in Measurements. Fuzzy Sets and Systems, 116, 141-154. https://doi.org/10.1016/S0165-0114(98)00280-2 http://www.sciencedirect.com/science/article/pii/S0165011498002802

[5] Ishikawa, S. (2006) Mathematical Foundations of Measurement Theory. Keio University Press Inc., Tokyo, 335 p. http://www.keio-up.co.jp/kup/mfomt/

[6] Ishikawa, S. (2019) Linguistic Copenhagen Interpretation of Quantum Mechanics; Quantum Language [Ver. 5]. Research Report, KSTS/RR-19/003, Department of Mathematics, Keio University, Yokohama, 473 p. http://www.math.keio.ac.jp/en/academic/research.html

[7] Ishikawa, S. (1991) Uncertainty Relation in Simultaneous Measurements for Arbitrary Observables. Reports on Mathematical Physics, 29, 257-273.

https://doi.org/10.1016/0034-4877(91)90046-P https://www.sciencedirect.com/science/article/abs/pii/003448779190046P

[8] Ishikawa, S. (2011) A New Interpretation of Quantum Mechanics. Journal of Quantum Information Science, 1, 35-42. https://doi.org/10.4236/jqis.2011.12005 
http://www.scirp.org/journal/PaperInformation.aspx?paperID=7610

[9] Ishikawa, S. (2015) Linguistic Interpretation of Quantum Mechanics; Projection Postulate. Journal of Quantum Information Science, 5, 150-155.

https://doi.org/10.4236/jqis.2015.54017

http://www.scirp.org/Journal/PaperInformation.aspx?PaperID=62464

[10] Ishikawa, S. (2017) Bell's Inequality Should Be Reconsidered in Quantum Language. Journal of Quantum Information Science, 7, 140-154.

https://doi.org/10.4236/jqis.2017.74011 http://www.scirp.org/Journal/PaperInformation.aspx?PaperID=80813

[11] Ishikawa, S. (2008) Dynamical Systems, Measurements, Quantitative Language and Zeno's Paradoxes. Far East Journal of Dynamical Systems, 10, 277-292.

http://www.pphmj.com/abstract/3595.htm

[12] Ishikawa, S. (2012) A Measurement Theoretical Foundation of Statistics. Applied Mathematics, 3, 283-292. https://doi.org/10.4236/am.2012.33044 http://www.scirp.org/journal/PaperInformation.aspx?paperID $=18109$ \&

[13] Ishikawa, S. (2012) Monty Hall Problem and the Principle of Equal Probability in Measurement Theory. Applied Mathematics, 3, 788-794.

https://doi.org/10.4236/am.2012.37117 http://www.scirp.org/journal/PaperInformation.aspx?PaperID=19884

[14] Ishikawa, S. (2012) Ergodic Hypothesis and Equilibrium Statistical Mechanics in the Quantum Mechanical World View. World Journal of Mechanics, 2, 125-130.

https://doi.org/10.4236/wjm.2012.22014

https://www.scirp.org/journal/paperinformation.aspx?paperid=18861

[15] Kikuchi, K. and Ishikawa, S. (2010) Psychological Tests in Measurement Theory. Far East Journal of Theoretical Statistics, 32, 81-99. http://www.pphmj.com/abstract/5006.htm

[16] Kikuchi, K. (2011) An Axiomatic Approach to Fisher's Maximum Likelihood Method. Nonlinear Studies, 2, 255-262.

[17] Ishikawa, S. (2012) Quantum Mechanics and the Philosophy of Language: Reconsideration of Traditional Philosophies. Journal of Quantum Information Science, 2, 2-9. https://doi.org/10.4236/jqis.2012.21002 http://www.scirp.org/journal/PaperInformation.aspx?paperID=18194

[18] Ishikawa, S. (2017) A Final Solution to Mind-Body Problem by Quantum Language, Journal of Quantum Information Science, 7, 48-56.

https://doi.org/10.4236/jqis.2017.72005

http://www.scirp.org/Journal/PaperInformation.aspx?PaperID=76391

[19] Ishikawa, S. (2018) Leibniz-Clarke Correspondence, Brain in a Vat, Five-Minute Hypothesis, McTaggart's Paradox, etc. Are Clarified in Quantum Language. Open Journal of Philosophy, 8, 466-480. https://doi.org/10.4236/ojpp.2018.85032 https://www.scirp.org/Journal/PaperInformation.aspx?PaperID=87862

[20] Ishikawa, S. (2019) Philosophy of Science for Scientists; The Probabilistic Interpretation of Science. Journal of Quantum Information Science, 9, 123-154. https://doi.org/10.4236/jqis.2019.93007 https://www.scirp.org/Journal/paperinformation.aspx?paperid=95447

[21] Ishikawa, S. (2020) Wittgenstein's Picture Theory in the Quantum Mechanical Worldview. Journal of Quantum Information Science, 10, 104-125, https://doi.org/10.4236/jqis.2020.104007 https://www.scirp.org/journal/paperinformation.aspx?paperid $=106233$

[22] Ishikawa, S. (2021) Fuzzy Logic in the Quantum Mechanical Worldview; Related to 
Zadeh, Wittgenstein, Moore, Saussure, Quine, Lewis Carroll, etc. Journal of Applied Mathematics and Physics, 9, 1583-1610. https://doi.org/10.4236/jamp.2021.97108 https://www.scirp.org/journal/paperinformation.aspx?paperid $=110830$

[23] Ishikawa, S. (2021) History of Western Philosophy from the Quantum Theoretical Point of View, Research Report [Ver.4]. KSTS-RR-21/001, Department of Mathematics, Keio University, Yokohama, 306 p. http://www.math.keio.ac.jp/en/academic/research.html

[24] Prior, N. (1968) Papers on Time and Tense. Oxford University Press, Oxford.

[25] Pnueli, A. (1977) The Temporal Logic of Programs. Proceedings of the 18th IEEE Symposium on Foundations of Computer Science, Providence, 31 October-2 Novemver 1977, 46-67 https://doi.org/10.1109/SFCS.1977.32

[26] von Neumann, J. (1932) Mathematical Foundations of Quantum Mechanics. Springer Verlag, Berlin.

[27] Sakai, S. (1971) $C^{*}$-Algebras and $W^{*}$-Algebras, Ergebnisse der Mathematik und ihrer Grenzgebiete (Band 60). Springer-Verlag, Berlin, Heidelberg, New York.

[28] Yosida, K. (1980) Functional Analysis. 6th Edition, Springer-Verlag, Heidelberg.

[29] Davies, E.B. (1976) Quantum Theory of Open Systems. Academic Press, Cambridge.

[30] Holevo, A.S. (1982) Probabilistic and Statistical Aspects of Quantum Theory. NorthHolland Publishing Company, Amsterdam.

[31] Wittgenstein, L. (1921) Tractatus Logico Philosophicus. Routledge and Kegan Paul, Oxford.

[32] Russell, B. (1921) The Analysis of Mind. George Allen and Unwin, London.

[33] Lewis, C.L. (1918) A Survey of Symbolic Logic. University of California, Oakland. 